\title{
Particle trapping at an advancing solidification front with interfacial-curvature effects
}

\author{
A.W. Rempel ${ }^{\mathrm{a}, *}$, M.G. Worster ${ }^{\mathrm{b}}$ \\ ${ }^{a}$ Applied Physics Laboratory, University of Washington, Box 355640, 1013 NE 40th Street, Seattle, WA 98105, USA \\ ${ }^{\mathrm{b}}$ Department of Applied Mathematics and Theoretical Physics, Institute of Theoretical Geophysics, University of Cambridge, \\ Silver Street, Cambridge CB3 9EW, UK
}

Received 1 October 2000; accepted 11 December 2000

Communicated by A.A. Chernov

\begin{abstract}
We predict the maximum solidification rate, or critical velocity, $V_{\mathrm{c}}$ at which an insoluble particle suspended in a melt is pushed ahead of an advancing solidification front by intermolecular forces. At higher solidification rates the particle is incorporated within the solid. The net intermolecular force pushing the particle and the viscous resistance opposing it are both significantly influenced by the shape of the front as it conforms to the particle in response to interfacial premelting. We predict the entire shape of the front, within a thin-film approximation, accounting for the freezing-point depression due to curvature. We show how the interface shape varies with the magnitude of the surface energy and the closeness of the particle, and compare these to previous, ad hoc representations of the interface. We confirm the scaling results of previous, more approximate, analyses for the case in which the intermolecular forces are dominated by nonretarded van der Waals interactions, and provide new results for other power-law interactions. We examine how the particle behaviour changes as its radius increases so that the effect of interfacial curvature is diminished. (C) 2001 Elsevier Science B.V. All rights reserved.
\end{abstract}

PACS: 64.70.Dv; 68.15.+e; 68.45.Gd; 81.30.Fb

Keywords: A1. Fluid flows; A1. Interfaces; A1. Particle trapping; A1. Premelting; A1. Solidification; A1. Surface processes

\section{Introduction}

An insoluble particle can be pushed ahead of an advancing solidification front if the solidification velocity is sufficiently slow, whereas it is trapped within the growing solid region if the solidification velocity is more rapid. The critical velocity $V_{\mathrm{c}}$ that

\footnotetext{
*Corresponding author. Tel.: +1-206-543-1274; fax: +1206-616-3142.

E-mail address: awrempel@apl.washington.edu (A.W. Rempel).
}

marks this change in behaviour is important for the fabrication of composites reinforced with ceramic particles [1], the casting of alloys, the cryogenic preservation of biological materials [2], and the formation of ice lenses in frozen soils [3]. Experiments show that $V_{\mathrm{c}}$ decreases (increases) as the particle radius $R$ becomes larger (smaller) [1,3-10], and the precise value of $V_{\mathrm{c}}$ depends on additional factors such as the properties of the particle's surface $[4,11,12]$ and the temperature gradient $G[5,6,8]$. Despite numerous attempts to model the interaction between a particle and a 
solidification front and predict the dependence of $V_{\text {c }}$ on $R$ and other parameters [1,8,9,13-20], no rigorous treatment of the capture of small particles has been produced. Most previous models have relied on ad hoc assumptions regarding such issues as the shape of the solid-liquid interface near the particle base and the thickness of the liquid film that separates them at the moment the particle is trapped. The shape of the solid-liquid interface is determined by the requirements of equilibrium in the vicinity of the particle base. We solve explicitly for the interface shape and calculate the force balance on the particle to show how both the film thickness and the value of $V_{\mathrm{c}}$ are controlled by the dynamics of the problem.

In an earlier contribution [20], we analysed the behaviour of a particle near an advancing solidification front for the case in which $R$ is sufficiently large (typically greater than about $10^{-4} \mathrm{~m}$ ) that, as the solid-liquid interface begins to deform around the particle's surface, its curvature remains small and has a negligible effect on the interface temperature. In this case, the equilibrium temperature of the interface depends only on its distance from the particle and the nature and strength of the dominant intermolecular interactions that cause this separation. The balance of forces on the particle indicates that $V_{\mathrm{c}} \propto R^{-1}$, regardless of the type of intermolecular interactions that dominate, whereas the dependence of $V_{\mathrm{c}}$ on the temperature gradient is different for different interaction types - for non-retarded van der Waals interactions $V_{\mathrm{c}} \propto G^{1 / 4}$, for example.

This paper is concerned with the case where $R$ is small enough that the influence of curvature on the equilibrium temperature significantly affects the geometry of the solid-liquid interface. In several previous models, the interface curvature has been assumed to take a constant value in the region near the base of the particle $[13,14,18]$; we show that this tends to underestimate the particleinterface separation at moderate lateral distances from the point of closest approach and leads to inaccurate predictions for the particle behaviour. A more appropriate approximation for the interface geometry was employed by Chernov and his colleagues $[15,16]$. These researchers analysed the behaviour of small particles under the assumption that the interface shape could be approximated to increasing degrees of accuracy, first with a paraboloid, then with the intersection of a paraboloid and a plane. In the current work, we solve explicitly for the entire interface shape. Initially, we focus on the case in which the intermolecular forces are dominated by non-retarded van der Waals interactions; the characteristic velocity scale that we determine agrees with the predictions of Chernov and his colleagues. We employ numerical techniques to predict the particle velocity as a function of its position relative to the undisturbed, planar isotherms. We discuss briefly how the particle behaviour changes when the repulsive force between the particle and the solid phase is generated by other types of intermolecular interactions. The transition between the behaviour of small and large particles is examined towards the end of the paper.

\section{The temperature at the solid-liquid interface}

The behaviour of an insoluble particle near an advancing solidification front is intimately connected to the effect that the particle's presence has on the equilibrium melting temperature of the solid as it approaches the particle base. At constant pressure, the melting of a bulk solid normally occurs at a single temperature $T_{\mathrm{m}}$. When the solid-liquid interface deviates from the plane, the melting temperature of a pure substance is reduced below $T_{\mathrm{m}}$ by an amount proportional to the curvature $\mathscr{K}$ and the surface energy $\sigma_{\text {sl }}$. This change in melting temperature is often referred to as the Gibbs-Thomson effect. Intermolecular interactions can cause the formation of melt between the solid and a substrate, even when the interface is planar so that $\mathscr{K} \equiv 0$. This phenomenon is referred to as interfacial premelting $[19,21-$ 26]. When interfacial premelting occurs, the interface temperature $T_{\mathrm{i}}$ is inversely related to the separation $d$ between the solid and the substrate so that

$$
T_{\mathrm{i}}=T_{\mathrm{m}}-\frac{T_{\mathrm{m}} \sigma_{\mathrm{sl}}}{\rho_{\mathrm{s}} q_{\mathrm{m}}} \mathscr{K}-T_{\mathrm{m}}\left(\frac{\lambda}{d}\right)^{v},
$$


where $\rho_{\mathrm{s}}$ and $q_{\mathrm{m}}$ are the solid density and the latent heat, and $\lambda$ is a length scale that characterises the strength of the intermolecular interactions that dominate (typically $\lambda$ is of order $10^{-10} \mathrm{~m}$ ). The exponent $v$ depends on the type of intermolecular forces that dominate; non-retarded van der Waals interactions are modelled using $v=3$, while values of 4 and 2 correspond to the cases of retarded van der Waals interactions and long-range electrical interactions, respectively [24]. The equilibrium undercooling $T_{\mathrm{m}}-T_{\mathrm{i}}$ increases as the film thickness is reduced, causing the solid-liquid interface to deviate from the plane of the $T_{\mathrm{m}}$ isotherm when it approaches an insoluble particle (see Fig. 1). The interfacial curvature that accompanies this deviation also affects the equilibrium undercooling. The interface geometry is key to determining the forces on the particle and predicting the particle behaviour.

We treat the case of a single, isolated particle in a temperature gradient $G$, with its centre a distance $H$ from the bulk-melting-temperature isotherm, as shown in Fig. 1. The form of Eq. (1) suggests that we define the length scales

$\Gamma \equiv \frac{T_{\mathrm{m}} \sigma_{\mathrm{sl}}}{\rho_{\mathrm{s}} q_{\mathrm{m}} R G}$ and

$l \equiv\left(\frac{\lambda^{v} T_{\mathrm{m}}}{G}\right)^{1 /(v+1)}$

to measure the importance of interfacial curvature and intermolecular interactions respectively. For the current geometry, the equilibrium condition on the interface temperature implies that

$\Gamma \kappa+\frac{l^{v+1}}{d^{v}}=(R+d) \cos \theta-H$,

where $\theta$ is the angle to the vertical and $\kappa \equiv R \mathscr{K}$ is the dimensionless curvature of the solidification front ( $\kappa$ can be related to $d$ by taking the divergence of the normal to the solidification front).

The right side of Eq. (3) is the vertical distance from the solidification front to the plane of the $T_{\mathrm{m}}$ isotherm; as such, it is directly proportional to the undercooling because the isotherms are assumed to remain horizontal. In practical circumstances, differing thermal conductivities can result in a distortion of the isotherms away from the horizontal [16]. We ignore these thermal effects in order to avoid obscuring the most basic physical balances that control the particle behaviour. The first term on the left side of Eq. (3) represents the

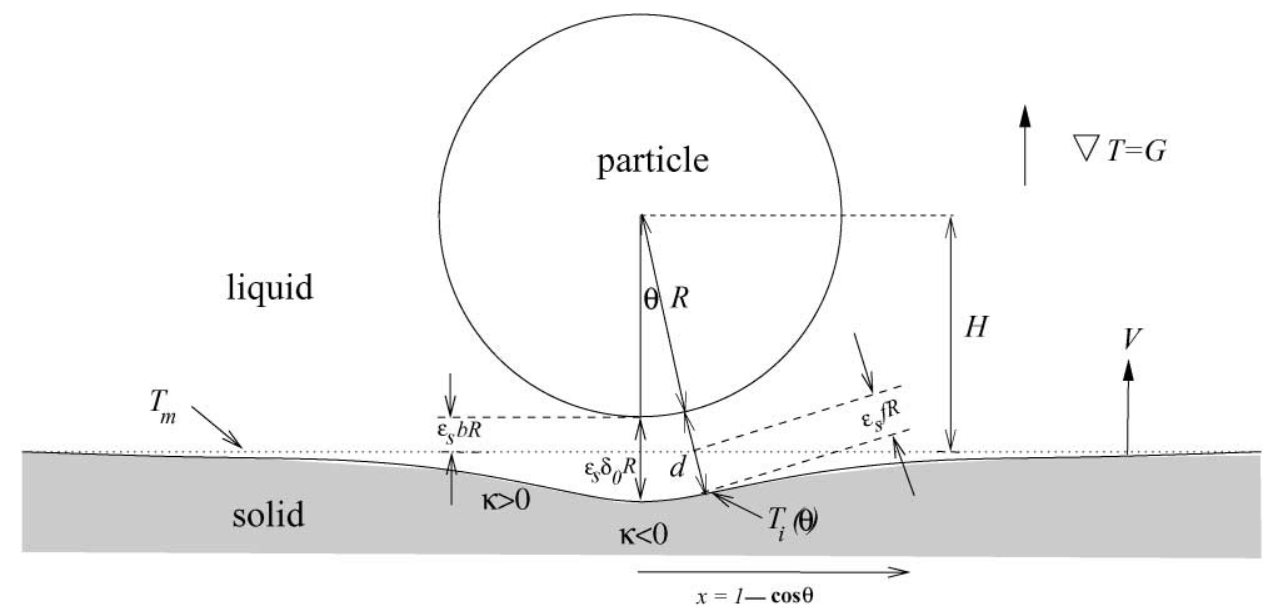

Fig. 1. A schematic diagram showing an insoluble particle suspended in a melt above an advancing solidification front. At large distances the solid-liquid interface conforms to the horizontal $T_{\mathrm{m}}$ isotherm. Near the particle base, the equilibrium interface temperature $T_{\mathrm{i}}$ is related to the curvature $\mathscr{K}$ and the thickness of the premelted film $d$. These vary with the angle $\theta$ for a given height $H$ of the particle centre above the $T_{\mathrm{m}}$ isotherm. 
effect of interfacial curvature. The second term on the left is the contribution due to intermolecular interactions. Boundary conditions consist of the symmetry condition at $\theta=0$ and the requirement that the solidification front follow the plane of the $T_{\mathrm{m}}$ isotherm far away from the particle so that

$\frac{\mathrm{d} d}{\mathrm{~d} \theta}=0 \quad$ at $\theta=0$

and

$d \rightarrow \frac{H-R \cos \theta}{\cos \theta}$ as $\theta \rightarrow \frac{\pi}{2}$.

We examine the magnitudes of the two terms on the left side of Eq. (3) to compare the relative importance of intermolecular interactions and interface curvature in controlling the interface temperature. As the particle nears the interface, $\kappa$ becomes of order unity and $d$ approaches $l$. We define the characteristic radius as the particle radius at which $\Gamma=l$, namely

$R_{\mathrm{c}}=\frac{\sigma_{\mathrm{sl}}}{\rho_{\mathrm{s}} q_{\mathrm{m}}}\left(\frac{T_{\mathrm{m}}}{\lambda G}\right)^{v /(v+1)}$.

For fixed $R$, Eq. (5) indicates that the effects of the interface curvature are more important (i.e. the ratio of $R / R_{\mathrm{c}}$ is smaller) when $G$ is lower and $\lambda$, which measures the strength of the dominant intermolecular interactions, is shorter. Curvature effects can be neglected when $R \gg R_{\mathrm{c}}$ and analytical expressions for $V_{\mathrm{c}}$ are readily derived [20]. We are interested here in cases where $R$ is either comparable to or much smaller than $R_{\mathrm{c}}$ so that the deformation of the solidification front significantly alters the equilibrium temperature and all of the terms in Eq. (3) are important. Typically, $R_{\mathrm{c}}$ is of order $10^{-4} \mathrm{~m}$. Table 1 contains a summary of parameter values for the case in which non-retarded van der Waals interactions dominate.

\section{The forces on a particle}

In addition to causing the formation of a premelted film, the intermolecular interactions between a solidification front and an insoluble particle generate the force per unit area $P_{\mathrm{T}}$ that repels the particle from the solid-liquid interface. It is worth noting that while Eq. (1) describes how both curvature effects and the proximity of the

Table 1

Typical parameter values for use in predicting the behaviour of an insoluble particle near an advancing solidification front ${ }^{\mathrm{a}}$

\begin{tabular}{|c|c|c|c|}
\hline & Parameter & Typical range & Nominal value \\
\hline \multirow[t]{3}{*}{ Experimental parameters } & $A_{\mathrm{sw}}$ & $10^{-20}-10^{-22} \mathrm{~J}$ & $10^{-20} \mathrm{~J}$ \\
\hline & $G$ & $1-10^{4} \mathrm{~K} \mathrm{~m}^{-1}$ & $10^{4} \mathrm{~K} \mathrm{~m}^{-1}$ \\
\hline & $R$ & $10^{-7}-10^{-4} \mathrm{~m}$ & $10^{-6} \mathrm{~m}$ \\
\hline \multirow[t]{5}{*}{ Physical constants for the ice-water system } & $\rho$ & $10^{3} \mathrm{~kg} \mathrm{~m}^{-3}$ & $10^{3} \mathrm{~kg} \mathrm{~m}^{-3}$ \\
\hline & $T_{\mathrm{m}}$ & $273.15 \mathrm{~K}$ & $273.15 \mathrm{~K}$ \\
\hline & $q_{\mathrm{m}}$ & $3.3 \times 10^{5} \mathrm{~J} \mathrm{~kg}^{-1}$ & $3.3 \times 10^{5} \mathrm{~J} \mathrm{~kg}^{-1}$ \\
\hline & $\sigma_{\mathrm{sl}}$ & $0.03 \mathrm{~J} \mathrm{~m}^{-2}$ & $0.03 \mathrm{~J} \mathrm{~m}^{-2}$ \\
\hline & $\mu$ & $1.8 \times 10^{-3} \mathrm{~Pa} \mathrm{~s}$ & $1.8 \times 10^{-3} \mathrm{~Pa} \mathrm{~s}$ \\
\hline \multirow[t]{2}{*}{ Dimensionless parameters } & $\gamma \equiv T_{\mathrm{m}} \sigma_{\mathrm{sl}} / \rho q_{\mathrm{m}} G R^{2}$ & $10^{-3}-10^{5}$ & 1 \\
\hline & $\varepsilon_{\mathrm{s}} \equiv\left(A_{\mathrm{sw}} / 6 \pi \sigma_{\mathrm{sl}} R^{2}\right)^{1 / 3}$ & $10^{-5}-10^{-2}$ & $10^{-3}$ \\
\hline \multirow[t]{2}{*}{ Characteristic scales } & $R_{\mathrm{c}} \equiv\left(6 \pi T_{\mathrm{m}}^{3} \sigma_{\mathrm{sl}}^{4} / \rho^{3} q_{\mathrm{m}}^{3} G^{3} A_{\mathrm{sW}}\right)^{1 / 4}$ & $10^{-4}-1 \mathrm{~m}$ & $10^{-4} \mathrm{~m}$ \\
\hline & $\mathscr{W} \equiv\left(\sigma_{\mathrm{sl}} A_{\mathrm{sw}}^{2} / 6^{5} \pi^{2} \mu^{3} R^{4}\right)^{1 / 3}$ & $10^{-9}-10^{-4} \mathrm{~m} \mathrm{~s}^{-1}$ & $10^{-5} \mathrm{~m} \mathrm{~s}^{-1}$ \\
\hline
\end{tabular}

\footnotetext{
${ }^{\mathrm{a}}$ The tabulated values for the effective Hamaker constant $A_{\text {sw }}$ are derived from the theoretical calculations of Ref. [27]. The nominal value of $10^{-20} \mathrm{~J}$ is comparable to that found for the interaction between ice and silicon through an intervening liquid film. In laboratory experiments, $G$ is often much larger than that normally experienced by soil particles in nature, for example. This, together with the large range in $R$, accounts for most of the variability in parameter values.
} 
particle cause $T_{\mathrm{i}}$ to change, thereby controlling the interface shape, it is only the intermolecular interactions between the particle and the solid, through the premelted film, that generate $P_{\mathrm{T}}$. To simplify the presentation, initially we assume that non-retarded van der Waals interactions are dominant so that $v=3$ and

$P_{\mathrm{T}}=\frac{\rho_{\mathrm{s}} q_{\mathrm{m}} \lambda^{3}}{d^{3}} \equiv \frac{A_{\mathrm{sw}}}{6 \pi d^{3}}$.

The second equality makes use of the definition of $\lambda$ in terms of the effective Hamaker constant $A_{\mathrm{sw}}$ for interactions between the particle wall and the solid, through the intervening premelted film (this is only valid when $v=3$ ). The effective Hamaker constant can be predicted from the dielectric response functions for the solid, the liquid and the particle [27], or it can be inferred from experiments $[23,24]$. The net thermomolecular force on the particle is found by integrating $P_{\mathrm{T}}$ over the interfacial surface. For a spherical particle near a solid-liquid interface, the net (vertical) thermomolecular force is

$$
\begin{aligned}
F_{\mathrm{T}} & =2 \pi R^{2} \int_{0}^{\theta_{\mathrm{c}}} \sin \theta \cos \theta P_{\mathrm{T}} \mathrm{d} \theta \\
& =2 \pi R^{2} \rho_{\mathrm{s}} q_{\mathrm{m}} \frac{G l^{4}}{T_{\mathrm{m}}} \int_{0}^{\theta_{\mathrm{c}}} \frac{\sin \theta \cos \theta}{d^{3}} \mathrm{~d} \theta
\end{aligned}
$$

where $d$ is a function of the angular position $\theta$. The film thickness increases rapidly with $\theta$ so that the integral in Eq. (7) is dominated by the region near the base of the particle and the precise choice of the integration limit $\theta_{\mathrm{c}}$ is not critical; for the numerical calculations that follow we use a limit that corresponds to $\theta_{\mathrm{c}} \rightarrow \pi / 2$.

The net thermomolecular force is balanced by the lubrication force associated with the fluid flow that is required to satisfy the mass balance. The hydrodynamic pressure $P_{1}$ in the liquid film is reduced from the pressure in the bulk melt that surrounds the remainder of the particle. A lubrication approximation (e.g. Ref. [28]) is used to relate the gradient in $P_{1}$ to the volume flux of fluid through the film near the particle base. The mass balance condition relates this volume flux to the particle velocity $U$. The net lubrication force is found by integrating $P_{1}$ over the interfacial surface to obtain [20]

$F_{\mu}=2 \pi \mu R^{4} U \int_{0}^{\theta_{\mathrm{c}}} \sin \theta \cos \theta\left(\int_{\theta_{\mathrm{c}}}^{\theta} \frac{\sin \phi}{d^{3}} \mathrm{~d} \phi\right) \mathrm{d} \theta$,

where the density difference between the solid and its melt have been neglected for simplicity. (Scaling arguments indicate that $F_{\mu}$ exceeds the drag force exerted by fluid flow around the bulk of the particle by a factor of order $d / R$.)

In the absence of any additional forces, $F_{\mathrm{T}}$ and $F_{\mu}$ must balance so that Eqs. (7) and (8) together determine the particle velocity as a function of its position relative to the horizontal isotherms. For scaling purposes, we can estimate the magnitudes of $F_{\mathrm{T}}$ and $F_{\mu}$ by evaluating Eqs. (7) and (8) using a characteristic film thickness $d_{0}$ and assuming that $\theta_{\text {c }}$ can be approximated by $\theta_{0} \ll 1$. This suggests that the forces scale like

$F_{\mathrm{T} 0}=\frac{2 \pi \rho q_{\mathrm{m}} G l^{4} R^{2}}{T_{\mathrm{m}}} \frac{\theta_{0}^{2}}{d_{0}^{3}}$

and

$F_{\mu 0}=12 \pi \mu R^{4} \frac{\mathscr{W} \theta_{0}^{4}}{d_{0}^{3}}$,

where $\mathscr{W}$ is a characteristic velocity scale. We have yet to determine appropriate values for $d_{0}$ and $\theta_{0}$, which are needed to define how $\mathscr{W}$ depends on the system parameters. To facilitate the analysis and motivate the choices for $d_{0}$ and $\theta_{0}$, it is useful to first rewrite Eq. (3) in non-dimensional form.

\section{Dimensionless equations}

The forces on the particle are dominated by the region where the premelted film is thinnest. Near $\theta=0$, the two terms on the left side of Eq. (3) are of opposite sign and their magnitudes are roughly balanced. We define the small parameter $\varepsilon_{\mathrm{s}}$ as

$\varepsilon_{\mathrm{s}} \equiv\left(\frac{l^{4}}{\Gamma R^{3}}\right)^{1 / 3}$.

We expect the film thickness to be of order $\varepsilon_{\mathrm{s}} R$ near the base of the particle and to diverge towards infinity as $\theta$ tends to $\pi / 2$, as dictated by the second boundary condition in Eq. (4). For physical intuition, with a micron-sized particle and 
$\varepsilon_{\mathrm{s}}$ of order $10^{-3}$, a typical film thickness at $\theta=0$ is a few nanometres. We scale the length scale $\Gamma$ by the particle radius so that $\Gamma \equiv \gamma R$. Initially, we focus on cases where $\gamma$ is much greater than $\varepsilon_{\mathrm{s}}$. Since $\varepsilon_{\mathrm{s}} / \gamma \equiv\left(R / R_{\mathrm{c}}\right)^{4 / 3}$, this condition is always satisfied for $R \ll R_{\mathrm{c}}$. Towards the end of this work, we look at the special case of medium-sized particles with $R$ comparable to $R_{\mathrm{c}}$ so that $\gamma$ and $\varepsilon_{\mathrm{S}}$ are roughly the same size (the analysis in Ref. [20] applies to the case in which $\varepsilon_{\mathrm{s}} \gg \gamma$ ).

The interface deformation is only significant at a small angular distance from the base of the particle. To take advantage of this, we define the film thickness perturbation as

$f=\frac{1}{\varepsilon_{\mathrm{S}} R}\left(d-\frac{H-R \cos \theta}{\cos \theta}\right)$.

Thus, $\varepsilon_{\mathrm{S}} R f$ is the radial distance from the position of the deformed solidification front to the $T_{\mathrm{m}}$ isotherm (see Fig. 1) and $f$ ranges from a value of order unity at the base of the particle to zero at large angular distances where the solid-liquid interface tends towards the plane.

The intermolecular interactions between the particle and the interface become significant when the particle base is close enough to the $T_{\mathrm{m}}$ isotherm that the deformation of the interface is comparable to $d$. This suggests that we focus on the particle behaviour at small values of $H-R$. We scale the particle position accordingly, using

$H=R+\varepsilon_{\mathrm{S}} R b$,

and concentrate on the dynamics when the reduced particle height $b$ is of order unity. We complete the variable transformation by defining the horizontal coordinate $x \equiv 1-\cos \theta$ so that $f$ is a positive, monotonically decreasing function of $x$, where $x$ ranges between 0 and 1 .

Eq. (3) for the film thickness can be transformed and written in terms of $f$ and $x$ as an expansion in powers of $\varepsilon_{\mathrm{s}}$. Near the base of the particle, intermolecular interactions provide a key contribution to the leading-order balance, whereas further away the curvature term roughly balances the right side of Eq. (3). To solve for the interface shape, we rewrite the leading-order balance from Eq. (3) in the inner region near the base of the particle where $x$ is of order $\varepsilon_{\mathrm{s}}$, and in the outer region far away from the particle where $x$ is of order unity.

\subsection{The outer region}

Away from the base of the particle the relatively large film thickness ensures that the effects of van der Waals interactions are negligible. When $x$ and $\gamma$ are of order unity the leading-order behaviour of Eq. (3) is described by

$$
\begin{aligned}
& {\left[(1-x)^{4}-(1-x)^{6}\right] f^{\prime \prime}-\left[3(1-x)^{3}-5(1-x)^{5}\right] f^{\prime}} \\
& \quad+\left[(1-x)^{2}-3(1-x)^{4}\right] f \approx \frac{f}{\gamma},
\end{aligned}
$$

where the derivatives are taken with respect $x$. The three terms on the left side of Eq. (13) represent the effects of curvature while the term on the right derives from the right side of Eq. (3), which is proportional to the undercooling. Van der Waals interactions are only important at much smaller values of $x$. The far-field boundary condition from Eq. (4) stipulates that $f$ tends to zero as $x$ tends to unity.

\subsection{The inner region}

In the inner region, where $x$ is of order $\varepsilon_{\mathrm{s}}$, to leading order in $\varepsilon_{\mathrm{s}}$ the equation for the film thickness perturbation is

$2 \xi f^{\prime \prime}+2 f^{\prime}+\frac{1}{(\xi+b+f)^{3}}=0$,

where the derivatives are taken with respect to $\xi \equiv \varepsilon_{\mathrm{s}}^{-1} x$. The first two terms in Eq. (14) describe the effects of curvature and the third term accounts for the intermolecular interactions between the particle and the solid; the undercooling contributes only a first-order correction to Eq. (14). The boundary condition at $x=0$ from Eq. (4) transforms to

$f^{\prime}+\frac{1}{2(b+f)^{3}}=0, \quad$ at $\xi=0$,

while the film thickness perturbation at $\xi=0$ is $f_{0}$.

Eqs. (13) and (14) are solved numerically to determine the interface shape, as described in the appendix. The force balance on the particle is then 
evaluated from Eqs. (7) and (8) to predict the particle behaviour.

\section{The particle velocity}

We are now in a position to choose a velocity scale for the problem by balancing the typical values $F_{\mathrm{T} 0}$ and $F_{\mu 0}$ from Eq. (9). The film thickness immediately beneath the particle is of order $\varepsilon_{\mathrm{s}} R$. The intermolecular interactions are most important in the inner region where $x$ is of order $\varepsilon_{\mathrm{s}}$, which suggests that $\theta_{0}$ is of order $\sqrt{\varepsilon_{\mathrm{s}}}$. Assigning these values to the parameters $d_{0}$ and $\theta_{0}$ in Eq. (9) and balancing these typical force scales enables us to define the velocity scale as

$\mathscr{W} \equiv \frac{\rho q_{\mathrm{m}} l^{4} G}{6 \mu T_{\mathrm{m}} R^{2} \varepsilon_{\mathrm{s}}}=\left(\frac{\sigma_{\mathrm{sl}} A_{\mathrm{sw}}^{2}}{6^{5} \pi^{2} \mu^{3} R^{4}}\right)^{1 / 3}$,

where the numerical factor is included for convenience. The velocity scale is proportional to $R^{-4 / 3}$ so larger particles are expected to experience a lower critical velocity and they will be trapped within the growing solid region more readily. It is interesting to note that $\mathscr{W}$ is independent of $G$.
The film thickness in the inner region is determined mainly by the balance between interfacial-curvature effects and non-retarded van der Waals interactions; the undercooling is absent from the leading-order balance. Since both the lubrication force and the thermomolecular force are strongest here, the particle velocity is insensitive to $G$. (This behaviour occurs because curvature effects cause a shift in the equilibrium melting temperature that has a larger magnitude than the undercooling.) The velocity scale given in Eq. (16) is identical to that proposed by Chernov and his colleagues $[15,16]$.

\subsection{Results}

Fig. 2. shows the profile of the solid-liquid interface near the base of the particle for several different values of $\gamma$. The horizontal distance from the base of the particle is scaled with $\sqrt{\varepsilon_{\mathrm{s}}} R$, while the vertical distance from the $T_{\mathrm{m}}$ isotherm is scaled with $\varepsilon_{\mathrm{s}} R$. For small values of $\gamma$ the solid-liquid interface abruptly relaxes onto the $T_{\mathrm{m}}$ isotherm. As $\gamma$ increases, curvature effects become more pronounced and the depression of the solid-liquid
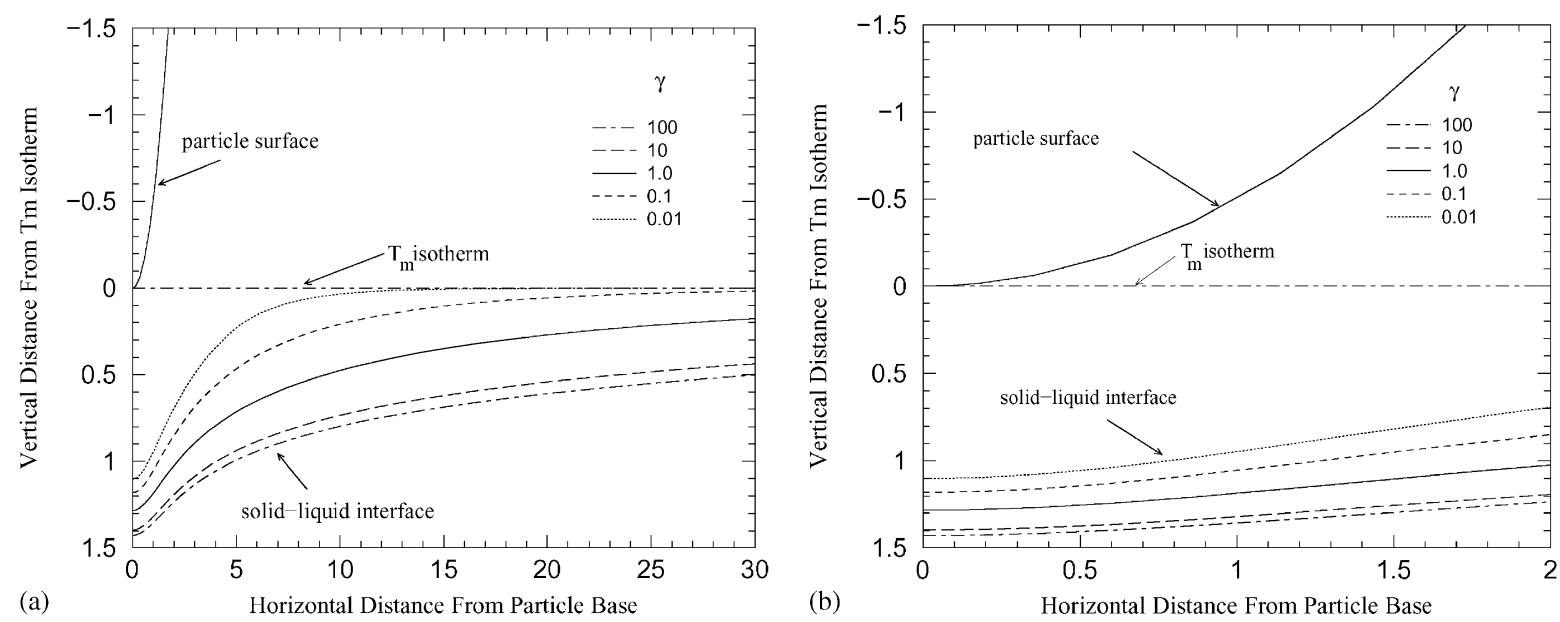

Fig. 2. The profile of the solid-liquid interface near the base of the particle for several different values of $\gamma$ when $\varepsilon_{\mathrm{S}}=10^{-3}$ and $b=0$. The horizontal coordinate is the distance from the base of the particle, scaled by $\sqrt{\varepsilon_{\mathrm{s}}} R$. The vertical coordinate is the distance below the $T_{\mathrm{m}}$ isotherm, scaled by $\varepsilon_{\mathrm{S}} R$. The surface of the particle is shown in the top-half of each image. Fig. 2a indicates that the solid-liquid interface relaxes onto the $T_{\mathrm{m}}$ isotherm more slowly for larger values of $\gamma$. The region near the base of the particle is magnified in (b). When the scaled radial distance is less than unity, the particle surface and the solid-liquid interface are relatively close together and roughly parallel. This is the region in which the forces on the particle are strongest. 
interface from the $T_{\mathrm{m}}$ isotherm changes more gradually with horizontal distance. The region near the base of the particle is magnified in Fig. 2b. When the horizontal distance from the base of the particle is less than order $\sqrt{\varepsilon_{\mathrm{s}}} R$, the solid-liquid interface has a slightly smaller curvature than that of the particle surface. Nevertheless, the normal to the interface is roughly perpendicular to the particle surface over this narrow region. This is where the dominant contributions to the forces on the particle originate, as we noted in choosing the value of $\theta_{0}$ for defining $\mathscr{W}$. Larger values of $\gamma$ are associated with a greater film thickness at all horizontal separations. Immediately beneath the particle this distance is of order $\varepsilon_{\mathrm{s}} R$, which provides justification for our choice of $d_{0}$.

In Fig. 3 we have plotted the dimensionless film thickness $\delta_{0} \equiv f_{0}+b$ as a function of the reduced particle height $b$ for several different values of $\gamma$. When $b$ is large and negative the edge of the particle is below the plane of the $T_{\mathrm{m}}$ isotherm. The solid-liquid interface begins to conform to the shape of the particle so that the dimensionless curvature at $x=0$ is roughly equal to -2 . The dimensionless film thickness tends to an asymptote of $\delta_{0} \sim 2^{-1 / 3}$, as shown by the horizontal line. When $b$ is large and positive the interactions

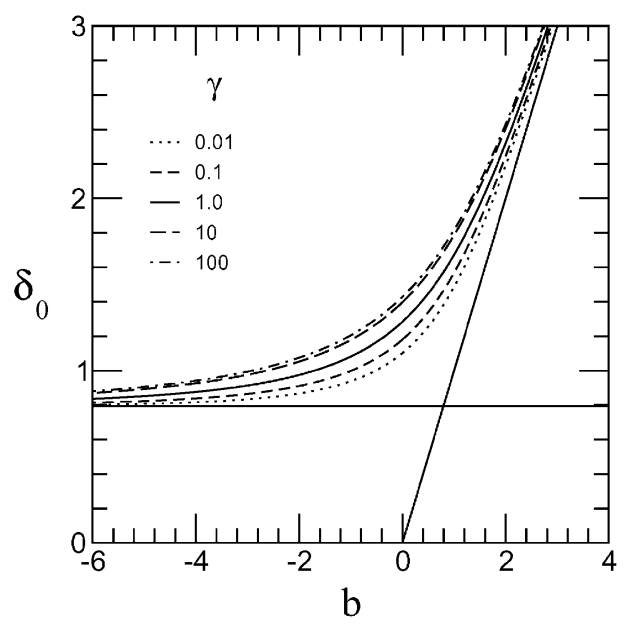

Fig. 3. The dimensionless film thickness $\delta_{0}$ as a function of the reduced particle height $b$ with $\varepsilon_{\mathrm{s}}=10^{-3}$. Each curve represents a different value of $\gamma$, as noted in the legend. between the particle and the solid are weak so the solid-liquid interface is almost planar. The film thickness perturbation $f_{0}$ tends to zero so that $\delta_{0} \approx b$ in this regime, as shown by the tendency of the curves to approach the solid line on the right side of the graph. When $b$ is near zero there is a transition in which the film thickness adjusts between the two asymptotes displayed on the graph. Smaller values of $\gamma$ correspond to a reduction in the effects of interfacial curvature and $\delta_{0}$ is able to relax more abruptly onto both asymptotes. When the effects of curvature are more pronounced and $\gamma$ is larger, the interface deflection is slightly greater at a given value of $b$.

The dimensionless particle velocity $u \equiv U / \mathscr{W}$ is shown as a function of the reduced particle height in Fig. 4. On the right side of the graph, where $b$ is large and positive, the dimensionless velocity is asymptotic to $b^{-1}$; this can be shown by

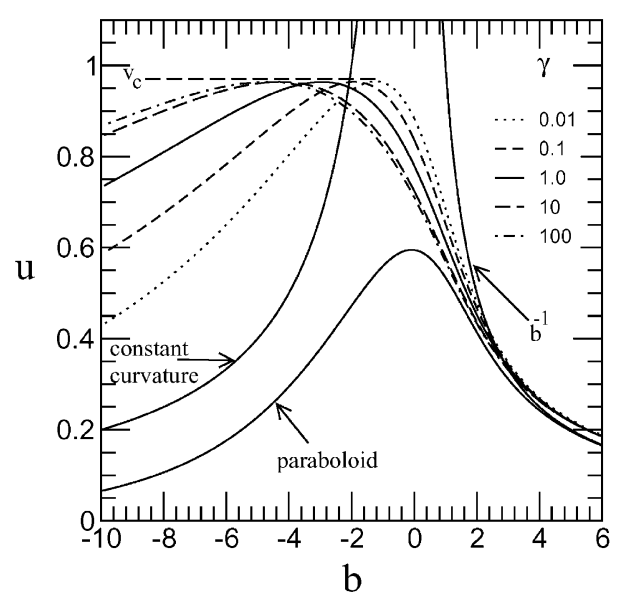

Fig. 4. The dimensionless particle velocity $u$ as a function of the reduced particle height $b$ with $\varepsilon_{\mathrm{s}}=10^{-3}$. Each curve represents a different vlaue of $\gamma$, as noted in the legend. Since $R / R_{\mathrm{c}} \equiv\left(\gamma / \varepsilon_{\mathrm{s}}\right)^{3 / 4}$, the different values of $\gamma$ may also be viewed as corresponding to different ratios of $R$ to $R_{\mathrm{c}}$. When $b \gg 0$ the interface is nearly planar and $u \approx b^{-1}$, as shown by the solid line on the right side of the graph. The velocity increases as $b$ decreases until it reaches a critical value of $v_{\mathrm{c}} \approx 1$. As $b$ decreases further, $u$ drops and the particle is trapped within the solid. The labelled solid lines on the left side of the graph and towards the bottom of the graph are found using different approximations for the interface geometry, as explained in the text. 
approximating the solid-liquid interface with a plane so that $f \equiv 0$ [29]. As the particle approaches the interface and $b$ decreases, the velocity of the particle increases until it either matches the solidification velocity or a critical velocity $v_{\mathrm{c}} \equiv V_{\mathrm{c}} / \mathscr{W}$ is reached. In the former case, an equilibrium separation is established between the particle and the interface; the particle will continue to travel ahead of the solidification front and remain nearly completely immersed in the bulk melt, as shown in Fig. 1. When the solidification velocity exceeds the critical velocity, however, the solidification front begins to encapsulate the particle and $b$ decreases further. The viscous resistance to fluid flow in the premelted film causes the particle velocity to decrease, as shown on the left side of the graph. This branch of the curve is unstable to finite perturbations of the particle position so the particle must become trapped within the solid region after $b$ decreases below the value that corresponds to the critical velocity $V_{\mathrm{c}}$. The critical velocity occurs at lower values of $b$ when curvature effects are stronger and $\gamma$ is larger. In Fig. 3 we showed that the film thickness is enhanced by larger values of $\gamma$. This causes the fluid flow to be less restricted and the particle is able to remain ahead of the advancing solidification front at lower values of $b$.

It is instructive to compare these predictions with those provided by various approximations for the interface shape. To obtain the solid curve on the left side of Fig. 4, we used a constant-curvature approximation in which the film thickness was calculated from Eq. (3) with $\kappa \equiv-2$ [29]. The graph demonstrates that this does not yield a very good approximation for $u$, particularly at larger values of $\gamma$. The reason for this can be deduced from further examination of Figs. 2 and 3. The dimensionless film thickness at $x=0$, shown in Fig. 3, tends rather quickly to the horizontal asymptote of $\delta_{0} \approx 2^{-1 / 3}$ when $b$ is negative. From Fig. 2b, however, it is apparent that, within a distance of order $\sqrt{\varepsilon_{\mathrm{s}}} R$ from the base of the particle, the solid-liquid interface has a less pronounced curvature than the particle surface. The constant-curvature approximation does not reflect this geometry and the film thickness is underestimated at small finite values of $x$. The solid curve towards the bottom of Fig. 4 shows the predictions of Chernov and his colleagues $[15,16]$ for the particle velocity. The calculation assumes that the shape of the solid-liquid interface near the base of the particle can be approximated by a paraboloid. Again the film thickness is underestimated away from the particle base and the predicted particle velocity is too low.

\section{Different interfacial interaction types}

The results presented thus far have been predicated on the assumption that non-retarded van der Waals forces control the thickness of the premelted film. Van der Waals forces are always present, but other forces sometimes dominate. Nevertheless, the dependence of the equilibrium melting temperature on the film thickness is often governed by a power law of the form described by Eq. (1). When this is the case, the theory presented above is easily modified by assigning the appropriate value to the exponent $v$, used in the definitions for the length scale $l$ in Eq. (2) and the characteristic radius $R_{\mathrm{c}}$ in Eq. (5). Long-range electrical interactions are modelled using $v=2$, while for retarded van der Waals interactions $v=4$ [24]. When the film thickness is only a few molecular diameters, steric effects can alter the equilibrium melting temperature so that it is not well described by the power-law relationships considered here [30]. When these effects dominate, the narrow film thickness will lead to very low critical velocities and the dynamics are of limited interest. Dissolved impurities that are present in the melt can also modify the thickness of the premelted film and double-layer forces can lead to an exponential relationship between the undercooling and the film thickness [31]. We do not consider such complications any further, though we recognise that they have the potential to exert a controlling influence on the behaviour of some experimental systems.

Once again, the particle velocity is found from the force balance on the particle. The lubrication force that tends to promote particle capture is still calculated using Eq. (8). The thermomolecular force must be found from the generalised form of 
Eq. (7), however, namely

$F_{\mathrm{T}}=2 \pi R^{2} \rho q_{\mathrm{m}} \frac{G l^{v+1}}{T_{\mathrm{m}}} \int_{0}^{\theta_{\mathrm{c}}} \frac{\sin \theta \cos \theta}{d^{v}} \mathrm{~d} \theta$.

The film thickness is obtained as the solution to Eq. (3) and the boundary conditions from Eqs. (4a) and (4b) remain unchanged.

The numerical calculations are performed after transforming the equations to nondimensional form in the same manner as before, but with the small parameter from Eq. (10) redefined as

$$
\varepsilon_{\mathrm{s}} \equiv\left(\frac{l^{v+1}}{\Gamma R^{v}}\right)^{1 / v}
$$

so that $\varepsilon_{\mathrm{s}} / \gamma \equiv\left(R / R_{\mathrm{c}}\right)^{(v+1) / v}$. This leaves the leading-order balance in the outer region unchanged from Eq. (13), in which terms representing the undercooling and the effects of curvature are included. In the inner region, Eq. (14) is generalised so that

$2 \xi f^{\prime \prime}+2 f^{\prime}+\frac{1}{(\xi+b+f)^{v}}=0$,

where the derivatives are taken with respect to $\xi \equiv$ $x / \varepsilon_{\mathrm{s}}$ and $\xi$ is of order unity. The boundary condition at $\xi=0$ becomes

$f^{\prime}+\frac{1}{2(b+f)^{v}}=0$.

The generalised velocity scale $\mathscr{W}$ is found by balancing typical values for the thermomolecular force and the lubrication force so that

$\mathscr{W} \equiv \frac{\sigma_{\mathrm{sl}} \lambda^{2}}{6 \mu R^{2}}\left(\frac{\rho q_{\mathrm{m}} R}{\sigma_{\mathrm{sl}}}\right)^{2 / v}$.

When long-range electrical interactions dominate so that $v=2$, this suggests that $V_{\mathrm{c}} \propto R^{-1}$. When $v=4$ and retarded van der Waals interactions dominate, $V_{\mathrm{c}} \propto R^{-3 / 2}$. These differences in the relationships between $V_{\mathrm{c}}$ and $R$ suggest a possible experimental approach for determining the type of intermolecular interactions that dominate in a given system. A survey of the published experimental data suggests that $V_{\mathrm{c}}$ is often proportional to $R^{-\alpha}$ where $\alpha$ is close to unity [20]. This is most consistent with our predictions when $v=2$, though a reasonable agreement with most of the data is also achieved when $v=3$. Note that the velocity scale is independent of the temperature gradient, as expected since the undercooling is absent from the leading-order balance in the inner region, described by Eq. (19). Sparse experimental data does not yield a consensus on the observed dependence of $V_{\mathrm{c}}$ on $G$ [20].

Fig. 5 shows the particle velocity as a function of the reduced particle height for different intermolecular interaction types. The parameter values were chosen so that the ratio of $R / R_{\mathrm{c}}$ is the same for each curve. (Note that the definition of $R_{\mathrm{c}}$ depends on the interaction type, as do the definitions of $\mathscr{W}$ and $\varepsilon_{\mathrm{s}}$, which must be used to transform to the dimensional equivalents of $u$ and $b$.) The solid line indicates that when $v=3$, the dimensionless critical velocity is roughly $u \approx 1$, as we saw in Fig. 4. The dotted line, corresponding to $v=4$, peaks at $u \approx 0.8$. When $v=2$ the dashed line seems to indicate that the particle velocity increases monotonically with increasing $b$. In the theory presented here the thermomolecular force is assumed to operate with a value of the exponent $v$ that is independent of the film thickness. This is reasonable for $v=3$ and 4 because $F_{\mathrm{T}}$ is dominated by the region immediately beneath the

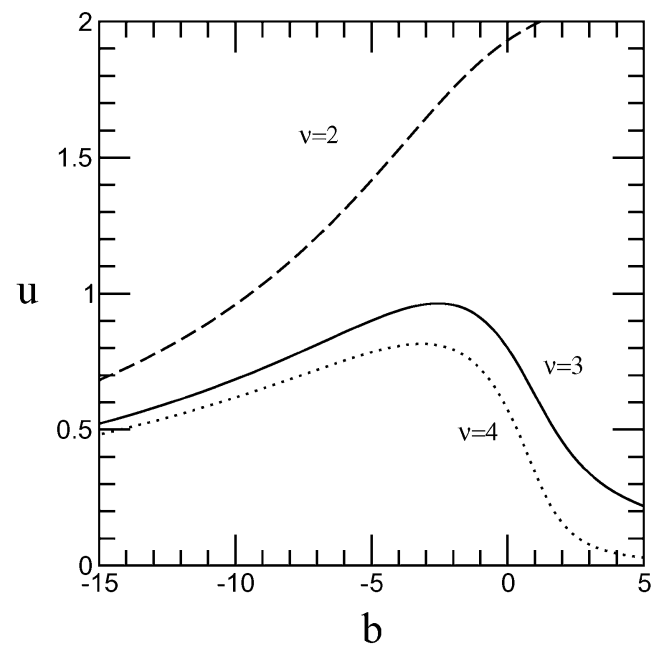

Fig. 5. The dimensionless particle velocity $u$ as a function of the reduced particle height $b$ with $\varepsilon_{\mathrm{s}}=10^{-3}$ and $R / R_{\mathrm{c}}=10^{-2}$. The curves represent the dynamics when different types of intermolecular interactions dominate. For $v=2$ the film thickness is controlled by long-range electrical interactions, for $v=3$ and 4 non-retarded and retarded van der Waals interactions dominate. 
particle where $d$ is thinnest. When $v=2$, however, the contributions to the force integral that are made at larger $d$ are overestimated. The predictions for $u$ with $b>0$ are not valid for $v=2$. Nevertheless, even for this case the critical velocity should remain a function of the velocity scale given in Eq. (21). Further analysis to predict the precise magnitude of $V_{\mathrm{c}}$ when $v=2$ is beyond the scope of the current investigation.

\section{Mid-sized particles}

For large particles, where $R \gg R_{\mathrm{c}}$ so that curvature effects are unimportant, $V_{\mathrm{c}} \propto R^{-1}$ [20]. When non-retarded van der Waals interactions dominate, $V_{\mathrm{c}}$ is also proportional to $G^{1 / 4}$ for $R \gg R_{\mathrm{c}}$ [20]. Here, we have shown that when $R \ll R_{\mathrm{c}}$ and $v=3$, $V_{\mathrm{c}} \propto R^{-4 / 3}$ and it is insensitive to $G$. To determine how particles of intermediate size behave entails investigating the parameter regime in which $\gamma$ is of order $\varepsilon_{\mathrm{s}}$ so that $R$ is close to $R_{\mathrm{c}}$, or equivalently the length scales $\Gamma$ and $l$ are comparable.

The analysis follows much the same procedure as we have devoted the early part of this paper to discussing. It is convenient for this case to use

$\varepsilon_{\mathrm{m}} \equiv \frac{l}{R}$,

as the small parameter, which is equal to $\varepsilon_{\mathrm{S}}$ when $R=R_{\mathrm{c}}$. Eq. (3) is non-dimensionalised by defining $f$ and $b$ as before, but with $\varepsilon_{\mathrm{m}}$ replacing $\varepsilon_{\mathrm{s}}$ in Eqs. (11) and (12). Since the length scale $\Gamma$ is comparable to $l$, we let

$\Gamma \equiv \varepsilon_{\mathrm{m}} g R$,

where $g$ is of order unity.

In the outer region, where $x$ is of order unity, to leading order in $\varepsilon_{\mathrm{m}}$ the equation for $f$ is trivial

$f=0$.

In the inner region where $x=\varepsilon_{\mathrm{m}} \xi$, with $\xi$ of order unity, to leading order in $\varepsilon_{\mathrm{m}}$ the governing equation is

$2 g \xi f^{\prime \prime}+2 g f^{\prime}+\frac{1}{(\xi+b+f)^{v}}=f$,

where $b$ is again taken to be of order unity. The first two terms in Eq. (25) derive from the effects of interfacial curvature, while the final term on the left is the contribution of intermolecular interactions between the particle and the solid. The undercooling is represented by the right side of the equation. Earlier we showed that, with $R \ll R_{\mathrm{c}}$, in the inner region $f$ is insensitive to the undercooling. When $R \gg R_{\mathrm{c}}$ curvature does not affect the interface position at all. In the intermediate case considered here, the effects of curvature, intermolecular interactions, and the undercooling all play a role in determining the film thickness in the inner region near the base of the particle.

To leading order in $\varepsilon_{\mathrm{m}}$, the boundary condition at $\xi=0$ is

$2 g f^{\prime}-f+\frac{1}{(b+f)^{v}}=0$.

The outer limit of the solution of Eq. (25) matches with the inner limit of the solution to Eq. (24), so $f \rightarrow 0$ as $\xi \rightarrow \infty$. The interface shape is determined from the equation for $f$ in the inner region alone. Eq. (25) was solved using a modified version of the numerical scheme discussed in the appendix.

In Fig. 6 we focus on the case in which nonretarded van der Waals interactions dominate so that $v=3$. Fig. 6 a shows the scaled critical velocity as a function of $g^{-1} \equiv R / R_{\mathrm{c}}$. On the left side of the graph, the tangent to the curve has a slope of $-\frac{4}{3}$. This is to be expected from the definition of $\mathscr{W}$ in Eq. (16), which is proportional to $R^{-4 / 3}$. On the right side of the graph, where interfacial curvature is less important, the tangent has a slope of -1 ; this is consistent with the analysis presented in Ref. [20]. In Fig. 6b we have plotted the scaled critical velocity as a function of $g^{-4 / 3}$, which is proportional to $G$. When $G$ is small, on the left side of the graph, $\Gamma>l$ so curvature effects are important and $V_{\mathrm{c}}$ is not sensitive to $G$. On the right side of the graph, where $\Gamma<l$, the interface deformation is less affected by curvature and $V_{\mathrm{c}} \propto G^{1 / 4}$, as shown by the dot-dashed asymptote.

\section{Conclusions}

We have shown how interfacial curvature affects the temperature of the solid-liquid interface near the base of a particle with a radius $R$ that is 

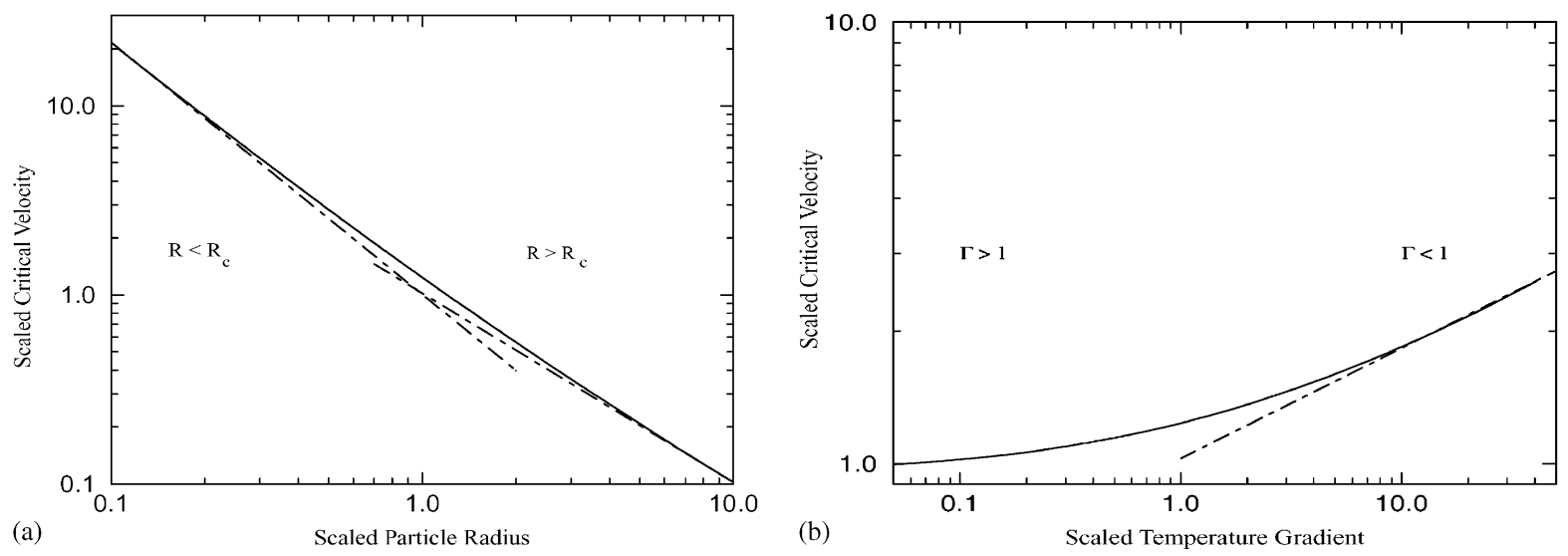

Fig. 6. The critical velocity for particles with $R$ comparable to $R_{\mathrm{c}}$. (a) $V_{\mathrm{c}}$ as a function of $R / R_{\mathrm{c}}$. $V_{\mathrm{c}}$ is scaled by $\mathscr{W} g^{-4 / 3}$, which is independent of $R$. On the left side of the graph, $V_{\mathrm{c}} \propto R^{-4 / 3}$, as shown by the slope of the tangent to the curve in this region. On the right side of the graph, where $R>R_{\mathrm{c}}$ the slope of the tangent indicates that $V_{\mathrm{c}} \propto R$. (b) $V_{\mathrm{c}}$ as a function of $G$, which is scaled so that $\Gamma=l$ when the horizontal coordinate is unity. $V_{\mathrm{c}}$ is scaled by $\mathscr{W}$ so that it is independent of $G$. On the left side of the graph, where the temperature gradient is low, the dynamics of the interactions between the particle and the solidification front are affected by the interface curvature. The critical velocity is insensitive to the temperature gradient in this regime. When $G$ is higher, $\Gamma<l$ and $V_{\mathrm{c}} \propto G^{1 / 4}$, as shown by the dot-dashed line on the right.

comparable to the characteristic radius $R_{\mathrm{c}}$, defined by Eq. (5). We used the calculated interface profile to evaluate the force balance and predict the particle behaviour. The particle is trapped within the solid if the solidification velocity is greater than some critical value $V_{\mathrm{c}}$. When the intermolecular interactions between the particle and the solidification front are dominated by non-retarded van der Waals interactions and $R \ll R_{\mathrm{c}}, \quad V_{\mathrm{c}} \propto R^{-4 / 3}$. In contrast to the case in which the interface curvature can be neglected, in this parameter regime, $V_{\mathrm{c}}$ is insensitive to the temperature gradient $G$ because the leadingorder balance is insensitive to the undercooling in the region where the forces on the particle are strongest. When other types of power-law interactions dominate the film thickness, $V_{\mathrm{c}}$ remains insensitive to $G$ but the dependence on $R$ changes; for retarded van der Waals interactions $V_{\mathrm{c}} \propto R^{-3 / 2}$, whereas, for long-range electrical interactions $V_{\mathrm{c}} \propto R^{-1}$. By examining the dynamics of particle trapping for the intermediate case in which $R$ is close to $R_{\mathrm{c}}$, we were able to illustrate the transition between the large and small particle regimes and show how the dependence of $V_{\mathrm{c}}$ on $R$ and $G$ changes as the influence of interfacial curvature is diminished.

\section{Acknowledgements}

Conversations with J.S. Wettlaufer are gratefully acknowledged.

\section{Appendix A. Numerical procedure}

A numerical procedure was adopted to determine $f$ from Eqs. (13) and (14). Given an initial estimate of the film thickness perturbation immediately beneath the particle $f_{0}$, a fourth-order Runga-Kutta integration routine with automatic step-size adjustment, adapted from Ref. [32], was used to calculate $f$ as a function of $x$. In implementing this integration routine, Eq. (14) from the inner region was used from $\xi_{1}=0$ to $\xi_{2}$, where $\varepsilon_{\mathrm{s}}^{-1}>\xi_{2}>1$. The value of $f$ and its first derivative at $\xi_{2}$ were then used as initial conditions for integrating Eq. (13) from $x_{1}=\varepsilon_{\mathrm{s}} \xi_{2}$ to $x_{2}$. Tests were performed to ensure that the results were insensitive to the value of $\xi_{2}$.

When the initial choice for $f_{0}$ was too large, the calculation for $f$ diverged to a large positive value for some $x_{2}<1$; this generated an upper bound on the actual value of $f_{0}$. When the initial choice for $f_{0}$ was too small, the calculation for $f$ diverged 
to a large negative number and a lower bound on the actual value of $f_{0}$ was established. A bisection algorithm was used repeatedly to revise the upper and lower bounds on $f_{0}$ until the difference between them was less than a predetermined tolerance $\Delta$. Typically, $\Delta$ was chosen to be of order $10^{-6} f_{0}$; tests were performed to ensure that the predictions were insensitive to further reduction in $\Delta$. This procedure enabled us to choose the value of $f_{0}$ that best approximates the far-field boundary condition, $f \rightarrow 0$ as $x \rightarrow 1$.

Once $f_{0}$ was determined, an integration routine was used to calculate scaled values for the thermomolecular force $f_{\mathrm{T}}$, the liquid pressure $p_{1}$ and the lubrication force $f_{\mu}$. These derive from Eqs. (7) and (8) so that

$\frac{\mathrm{d} f_{\mathrm{T}}}{\mathrm{d} x}=\frac{(1-x)^{4}}{\left(x+\varepsilon_{\mathrm{s}}(b+f)\right)^{3}}$,

$\frac{\mathrm{d} p_{1}}{\mathrm{~d} x}=\frac{(1-x)^{3}}{\left(x+\varepsilon_{\mathrm{s}}(b+f)\right)^{3}}$,

and

$\frac{\mathrm{d} f_{\mu}}{\mathrm{d} x}=(1-x) p_{1}$

subject to the boundary conditions

$f_{\mathrm{T}}=0=f_{\mu} \quad$ at $x=0$

and

$p_{1}=0 \quad$ at $x=x_{2}$.

The dimensionless particle velocity was then calculated as

$u \equiv \frac{U}{\mathscr{W}}=\varepsilon_{\mathrm{s}} \frac{f_{\mathrm{T}}}{f_{\mu}}$,

where $f_{\mathrm{T}}$ and $f_{\mu}$ were evaluated at $x=x_{2}$, and the velocity scale $\mathscr{W}$ is defined in Eq. (16).

\section{References}

[1] D. Shangguan, S. Ahuje, D.M. Stefanescu, Met. Trans. A 23A (1992) 669.

[2] V.L. Bronstein, Y.A. Itkin, G.S. Ishkov, J. Crystal Growth 52 (1981) 345.
[3] A.E. Corte, J. Geophys. Res. 67 (1962) 1085.

[4] J. Cissé, G.F. Bolling, J. Crystal Growth 10 (1971) 67.

[5] C. Korber, G. Rau, M.D. Cosman, E.G. Carvalho, J. Crystal Growth 72 (1985) 649.

[6] G. Lipp, C. Korber, G. Rau, J. Crystal Growth 99 (1990) 206.

[7] M.A. Azouni, W. Kalita, M. Yemmou, J. Crystal Growth 99 (1990) 201.

[8] G. Lipp, C. Korber, J. Crystal Growth 130 (1993) 475.

[9] P. Casses, M.A. Azouni, Int. Commun. Heat Mass Transfer 22 (1995) 605.

[10] S. Sen, B.K. Dhindaw, D.M. Stefanescu, A. Catalina, P.A. Curreri, J. Crystal Growth 173 (1997) 574.

[11] S.N. Omenyi, A.W. Neumann, J. Appl. Phys. 47 (1976) 3956.

[12] M.A. Azouni, P. Casses, B. Sergiani, Colloids Surf. A 122 (1997) 199.

[13] D.R. Uhlmann, B. Chalmers, K.A. Jackson, J. Appl. Phys. 35 (1964) 2986.

[14] G.F. Bolling, J. Cissé, J. Crystal Growth 10 (1971) 56.

[15] A.A. Chernov, D.E. Temkin, A.M. Mel'nikova, Sov. Phys. Crystallogr. 21 (1976) 369.

[16] A.A. Chernov, D.E. Temkin, in: E. Kaldis, H.J. Scheel (Eds.), Current Topics in Materials Science, Vol. 2, 1976, Crystal Growth and Materials, North-Holland, Amsterdam, 1977, p. 3.

[17] R.R. Gilpin, J. Colloid Interface Sci. 74 (1980) 44.

[18] J. Potschke, V. Rogge, J. Crystal Growth 94 (1989) 726.

[19] M.G. Worster, J.S. Wettlaufer, in: W. Shyy (Ed.), Fluid Dynamics at Interfaces, Cambridge University Press, Cambridge, 1999, p. 339.

[20] A.W. Rempel, M.G. Worster, J. Crystal Growth 205 (1999) 427.

[21] J.G. Dash, Contemp. Phys. 30 (1989) 89.

[22] D.W. Oxtoby, in: J.S. Wettlaufer, J.G. Dash, N. Untersteiner (Eds.), Ice Physics and the Natural Environment, Springer, New York, 1999, p. 23.

[23] J.G. Dash, H. Fu, J.S. Wettlaufer, Rep. Prog. Phys. 58 (1995) 115.

[24] J.S. Wettlaufer, M.G. Worster, L.A. Wilen, J.G. Dash, Phys. Rev. Lett. 76 (1996) 3602.

[25] J.S. Wettlaufer, Philos. Trans. Roy. Soc. Lond. A 357 (1999) 3403.

[26] J.S. Wettlaufer, J.G. Dash, Sci. Am. 282 (2000) 56.

[27] L.A. Wilen, J.S. Wettlaufer, M. Elbaum, M. Schick, Phys. Rev. B 52 (1995) 12426.

[28] G.K. Batchelor, An Introduction to Fluid Dynamics, Cambridge University Press, Cambridge, 1967, pp. 615

[29] A.W. Rempel, Ph.D. Thesis, University of Cambridge, 2000, pp. 226.

[30] J. Israelachvili, Intermolecular and Surface Forces, Academic Press, London, 1992, pp. 450.

[31] J.S. Wettlaufer, Phys. Rev. Lett. 82 (1999) 2516.

[32] W.H. Press, S.A. Teukolsky, W.T. Vetterling, B.P. Flannery (Eds.), Numerical Recipes in FORTRAN: The Art of Scientific Computing, 2nd edition, Cambridge University Press, Cambridge, 1992, pp. 963. 